\title{
Propagation of a whistler wave incident from above on the lower nighttime ionosphere
}

\author{
Peter Bespalov ${ }^{1,2}$ and Vera Mizonova ${ }^{3}$ \\ ${ }^{1}$ Institute of Applied Physics RAS, Nizhny Novgorod, Russia \\ ${ }^{2}$ National Research University Higher School of Economics, Nizhny Novgorod, Russia \\ ${ }^{3}$ State Technical University, Nizhny Novgorod, Russia \\ Correspondence to: Peter Bespalov (pbespalov@mail.ru)
}

Received: 3 March 2017 - Revised: 13 April 2017 - Accepted: 21 April 2017 - Published: 22 May 2017

\begin{abstract}
The problems of reflection and transmission of a whistler wave incident in the nighttime ionosphere from above are considered. Numerical solution of the wave equations for a typical condition of the lower ionosphere is found. The solution area comprises both the region of strong wave refraction and a sharp boundary of the nighttime ionosphere ( $\sim 100 \mathrm{~km}$ ). The energy reflection coefficient and horizontal wave magnetic field on the ground surface are calculated. The results obtained are important for analysis of the extremely low-frequency and very low-frequency (ELF-VLF) emission phenomena observed from both the satellites and the ground-based observatories.
\end{abstract}

Keywords. Magnetospheric physics (magnetosphereionosphere interactions; plasma waves and instabilities)

\section{Introduction}

Whistler propagation in the ionosphere has been the subject of many studies (e.g. Storey, 1953; Helliwell, 1965; Pitteway and Jespersen, 1966; Galejs, 1972; Budden, 1985; Yagitani et al., 1994; Lehtinen and Inan, 2008; Kuzichev and Shk1yar, 2010). Methods for finding wave fields depend on the ionosphere conditions and can be different for different altitude ranges. Above approximately $100 \mathrm{~km}$, the scale of the plasma inhomogeneity is on the order of $100 \mathrm{~km}$, which significantly exceeds the whistler wavelength. This permits using approximate methods, for example, geometrical optics equations (Ginzburg, 1970). At altitudes close to $100 \mathrm{~km}$ and below, the scale of the plasma inhomogeneity in the nighttime ionosphere is on the order of one or several kilometres. In addition, the wavelength of the wave propagating towards the Earth's surface increases, the wave attenuation due to charged and neutral particle collisions is enhanced, and the whistler wave transforms to a vacuum electromagnetic wave. In this case, approximate methods become unsuitable. Approximate methods are also unsuitable in the area of strong wave refraction. Thus, finding the field of an electromagnetic wave of the whistler frequency band in the lower layers of the ionosphere requires complete solution of the wave equations.

In this paper, we use numerical methods to find the field of a whistler wave incident from above in the lower layers of the ionosphere. One of the difficulties is associated with the divergence caused by the wave solutions with a large imaginary component of the vertical wave number ( $\mathrm{Ny}$ gre'n, 1982; Budden, 1985; Lehtinen and Inan, 2008). This problem was partially resolved in the paper by Lehtinen and Inan (2008), using the idea of recursive calculation of reflection coefficients. Kuzichev and Shklyar (2010) removed the divergence analytically and reduced numerical calculations to stable equations for the case of a sufficiently small angle of incidence. We use a technique known as the boundaryvalue problem. The theoretical approach to boundary-value problems is based on the solution of initial-value problems for ordinary differential equations and the solution of nonlinear algebraic equations using a shooting method. Using this technique provides numerically stable solutions of the complete system of wave equations in the ionosphere. For typical nighttime geophysical conditions, we calculate the energy reflection coefficient from the ionosphere and the horizontal wave magnetic field on the ground surface. The obtained results are important for analysis of the ELF-VLF emission phenomena observed from both the satellites and the groundbased observatories. 


\section{Problem formulation and basic equations}

We consider an incident of a whistler wave with frequency $\omega$ and wave vector $\boldsymbol{k}$ from above on the surface $z=z_{*}$. We suppose that the ambient magnetic field $\boldsymbol{B}$ belongs to the plane $y z$ and is inclined at angle $\vartheta$ to the $z$ axis; the coordinate $z=0$ corresponds to the ground surface, the plasma density depends on the vertical coordinate $z$, and for $z>z_{*}$ the plasma is close to homogeneous. The ionosphere can be considered as a stratified anisotropic cool plasma with the permittivity tensor (Shafranov, 1967)

$\hat{\boldsymbol{\varepsilon}}=\left(\begin{array}{ccc}\varepsilon & -i g \cos \vartheta & i g \sin \vartheta \\ i g \cos \vartheta & \varepsilon \cos ^{2} \vartheta+\eta \sin ^{2} \vartheta & (\eta-\varepsilon) \sin \vartheta \cos \vartheta \\ -i g \sin \vartheta & (\eta-\varepsilon) \sin \vartheta \cos \vartheta & \varepsilon \sin ^{2} \vartheta+\eta \cos ^{2} \vartheta\end{array}\right)$.

Here

$$
\begin{aligned}
& \varepsilon=1-\frac{\omega_{p_{e}}^{2}\left(\omega+i \nu_{e}\right)}{\omega\left(\left(\omega+i v_{e}\right)^{2}-\omega_{B_{e}}^{2}\right)}-\frac{\omega_{p_{i}}^{2}\left(\omega+i \nu_{i}\right)}{\omega\left(\left(\omega+i \nu_{i}\right)^{2}-\omega_{B_{i}}^{2}\right)}, \\
& \eta=1-\frac{\omega_{p_{e}}^{2}}{\omega\left(\omega+i \nu_{e}\right)}-\frac{\omega_{p_{i}}^{2}}{\omega\left(\omega+i v_{i}\right)}, \\
& g=-\frac{\omega_{p_{e}}^{2} \omega_{B_{e}}}{\omega\left(\left(\omega+i v_{e}\right)^{2}-\omega_{B_{e}}^{2}\right)}+\frac{\omega_{p_{i}}^{2} \omega_{B_{i}}}{\omega\left(\left(\omega+i v_{i}\right)^{2}-\omega_{B_{i}}^{2}\right)},
\end{aligned}
$$

where $\omega_{p_{e}}^{2}=4 \pi e^{2} n(z) / m_{e}$ is the square of the electron plasma frequency, $\omega_{p_{i}}^{2}=4 \pi Z^{2} e^{2} n(z) / m_{i}$ is the square of the ion plasma frequency, $Z$ is the dominant ion charge number, $v_{e, i}$ are the electron-neutral and the ion-neutral collision frequencies, $\omega_{B_{e}}=|e| B / m_{e} c$ and $\omega_{B_{i}}=\left|Z_{e}\right| B / m_{i} c$ are the values of the electron and ion gyrofrequencies, and $m_{e, i}$ are the electron and ion masses. Using the Maxwell equations

$\operatorname{rot} \boldsymbol{H}=-i \frac{\omega}{c} \hat{\boldsymbol{\varepsilon}} \boldsymbol{E}, \quad \operatorname{rot} \boldsymbol{E}=i \frac{\omega}{c} \boldsymbol{H}$

after substituting the wave electric and magnetic fields as $\boldsymbol{E}(z) \exp i\left(k_{x} x+k_{y} y-\omega t\right)$ and $\boldsymbol{H}(z) \exp i\left(k_{x} x+k_{y} y-\omega t\right)$, we find the following system for the transversal components $E_{x}, E_{y}, H_{x}, H_{y}$ :

$\frac{\mathrm{d} \boldsymbol{F}}{\mathrm{d} z}=\hat{\mathbf{M}} \boldsymbol{F}$

Here is a four-component vector column

$\boldsymbol{F}=\left(\begin{array}{l}E_{x} \\ E_{y} \\ (\omega / c) H_{x} \\ (\omega / c) H_{y}\end{array}\right)$

Vertical components of the wave electric and magnetic fields $E_{z}, H_{z}$ in accordance with Eq. (3) are expressed by means of the relationships

$$
\begin{aligned}
& E_{z}=\cot \vartheta E_{y}+(i / g \sin \vartheta)\left(\varepsilon E_{x}+(c / \omega)\left(k_{x} H_{y}-k_{y} H_{x}\right)\right), \\
& H_{z}=(c / \omega)\left(k_{x} E_{y}-k_{y} E_{x}\right) .
\end{aligned}
$$

The elements of matrix $\hat{\mathbf{M}}$ depend on the coordinate $z$ and are equal to

$$
\begin{aligned}
& m_{11}=-m_{44}=-k_{x}\left(g / \varepsilon_{z z}\right) \sin \vartheta \\
& m_{12}=-m_{34}=-i k_{x}\left((\eta-\varepsilon) / \varepsilon_{z z}\right) \sin \vartheta \cos \vartheta \\
& m_{13}=-m_{24}=-i\left(k_{x} k_{y} c^{2} / \omega^{2} \varepsilon_{z z}\right), \\
& m_{14}=-i-\left(k_{x} / k_{y}\right) m_{13} \\
& m_{21}=m_{43}=\left(k_{y} / k_{x}\right) m_{11}, \quad m_{22}=m_{33}=\left(k_{y} / k_{x}\right) m_{12}, \\
& m_{23}=i+\left(k_{y} / k_{x}\right) m_{13}, \\
& m_{31}=i k_{x} k_{y}-\left(\omega^{2} g \eta / c^{2} \varepsilon_{z z}\right) \cos \vartheta, \\
& m_{32}=-i k_{x}^{2}+i\left(\omega^{2} \varepsilon \eta / c^{2} \varepsilon_{z z}\right) \\
& m_{41}=i k_{y}^{2}-i\left(\omega^{2} \varepsilon / c^{2}\right)+i\left(\omega^{2} g^{2} / c^{2} \varepsilon_{z z}\right) \sin ^{2} \vartheta, \\
& m_{42}=m_{31}-2 i k_{x} k_{y},
\end{aligned}
$$

where $\varepsilon_{z z}=\varepsilon \sin ^{2} \vartheta+\eta \cos ^{2} \vartheta$.

To find the wave field, the solution for the system of Eqs. (4)-(6) must satisfy four boundary conditions. Assuming the ground surface $z=0$ to be perfectly conductive, we can write two of them:

$E_{x}(z=0)=0, \quad E_{y}(z=0)=0$.

To determine two other conditions, we express the field vector $\boldsymbol{F}$ at the upper boundary $z=z_{*}$ as the sum of four wave solutions corresponding to four local roots $\kappa_{j} \equiv k_{z j}$ of the dispersion relation $\boldsymbol{F}=\sum_{j=1}^{4} \boldsymbol{f}_{j} \exp \left(\int_{z_{*}}^{z} i \kappa_{j} \mathrm{~d} z^{\prime}\right), \hat{\mathbf{M}} \boldsymbol{f}_{j}=$ $i \kappa_{j} \boldsymbol{f}_{j}$. In this case,

$\boldsymbol{F}=\hat{\mathbf{P}} \boldsymbol{A}$.

Here, $A_{j}=A_{0 j} \exp \left(\int_{z^{*}}^{z} i \kappa_{j} \mathrm{~d} z^{\prime}\right)$ shows the amplitudes of the waves $(j=1-4)$ at the upper boundary $z=z_{*}, i \kappa_{j}$ are the eigenvalues of the matrix $\hat{\mathbf{M}}$, and the polarization matrix $\hat{\mathbf{P}}$ is formed by normalized eigenvectors of the matrix $\hat{\mathbf{M}}$ : $P_{q j}=\left(1 / \alpha_{j}\right) f_{q j}$, where $\alpha_{j}^{2}=\sum_{q=1}^{4}\left|f_{q j}\right|^{2}$. We assume that the first root corresponds to the incident wave (propagating oppositely to the $z$ axis), the second root corresponds to the reflected wave (propagating in the direction of the $z$ axis), and the third and fourth roots correspond to the exponentially increasing and exponentially decreasing waves in the $z$-axis direction. Then, two other boundary conditions can be written as

$A_{1}\left(z=z_{*}\right)=1, \quad A_{3}\left(z=z_{*}\right)=0$,

where $\boldsymbol{A}=\hat{\mathbf{P}}^{-1} \boldsymbol{F}$. The first condition (Eq. 9) determines the field of the incident wave, and the second condition (Eq. 9) excludes the exponentially increasing wave solution in the region $z>z_{*}$. 


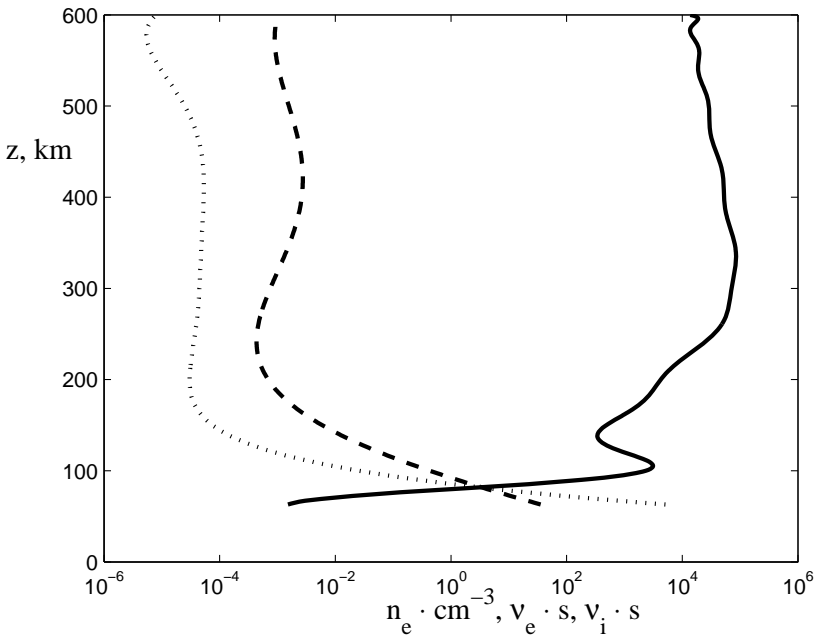

Figure 1. The plasma density (solid), the electron-neutral collision frequency (dashed), and the ion-neutral (dotted) collision frequency under conditions of the nighttime ionosphere.

Solving the system (Eqs. 4-6) in the range $0 \leq z \leq z_{*}$, we can obtain altitude dependencies of the wave electric and magnetic fields and the energy reflection coefficient from the boundary $z=z_{*}$

$R=\frac{\left|S_{z 2}\right|}{\left|S_{z 1}\right|}=\left.\frac{\left|A_{2}\right|^{2}}{\left|A_{1}\right|^{2}} \frac{\left|P_{12} P_{42}^{*}-P_{22} P_{32}^{*}\right|}{\left|P_{11} P_{41}^{*}-P_{21} P_{31}^{*}\right|}\right|_{z=z_{*}}$,

where $S_{z 1,2}$ are the vertical components of the Poynting vector of the wave incident on the boundary $z=z_{*}$ and reflected from this boundary, respectively.

\section{Typical calculation results}

We now present results of numerical solution of the system (Eqs. 4-6) with boundary conditions (Eqs. 7 and 9) under conditions typical of the lower nighttime ionosphere. As the basis we use the altitude dependencies of plasma density and the electron-neutral and ion-neutral collision frequencies sketched in Fig. 1. The dependencies of plasma density are taken from International Reference Ionosphere 2007 (Bilitza and Reinisch, 2008) and correspond to $67^{\circ} \mathrm{N}, 26^{\circ} \mathrm{E}$; $\vartheta=168^{\circ} ; 1$ January 2017; and 00:30 h LT.

To obtain the solution of system (Eqs. 4-6) in the region $0 \leq z \leq z_{*}$ with boundary conditions (Eqs. 7 and 9), we use a numerical method known as the two-point boundary-value problem for ordinary differential equations. For each frequency range, we choose the value $z_{*}$ such that for $z_{*} \leq z$, it satisfies the inequalities

$\left|\Delta z \operatorname{Re}\left(\kappa_{1,2}\right)\right| \gg 1, \quad\left|\Delta z \operatorname{Im}\left(\kappa_{3,4}\right)\right| \gg 1$,

where $\Delta z$ is the special scale of plasma inhomogeneity. Thus, the solution area covers a region that requires complete solu-

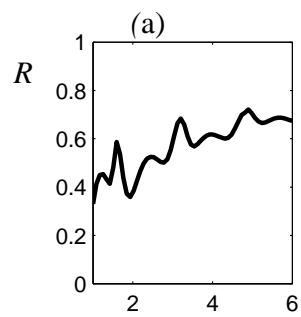

(b)

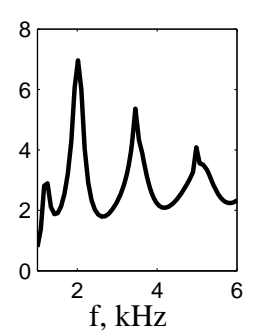

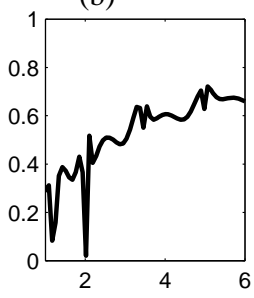

(c)
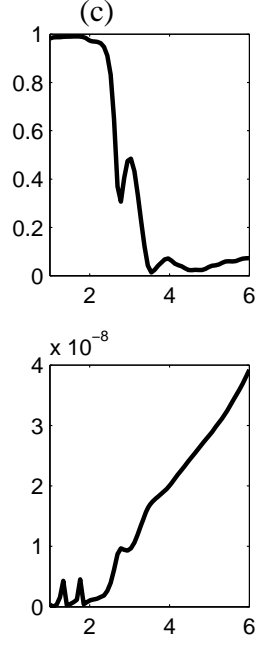

Figure 2. The energy reflection coefficient $R$ and the normalized amplitude of the horizontal magnetic field of the wave on the ground surface $h$ : (a) $k_{x}=k_{y}=0, z_{*}=125 \mathrm{~km}$; (b) $k_{x}=0.02 \mathrm{~km}^{-1}, k_{y}=$ $-0.02 \mathrm{~km}^{-1}, z_{*}=125 \mathrm{~km}$; and (c) $k_{x}=0 \mathrm{~km}^{-1}, k_{y}=0.3 \mathrm{~km}^{-1}$, $z_{*}=220 \mathrm{~km}$.

tion of the wave equations. Above boundary $z=z_{*}$ the geometrical optics equations (Ginzburg, 1970) can be useful.

Frequency and angular dependencies of the energy reflection coefficient $R$ and the normalized amplitude of the horizontal magnetic field $h$ of the wave on the ground surface are shown in Fig. 2. The horizontal wave magnetic field is usually measured on the ground surface, and the wave electric field is usually measured onboard the satellites above the ionosphere. Thus, the amplitude of the horizontal wave magnetic field on the ground surface is normalized by the electric field amplitude of the incident wave

$h=\left(\frac{\left.\left(\left|H_{x}\right|^{2}+\left|H_{y}\right|^{2}\right)\right|_{z=0}}{\left.\left(\left|E_{i x}\right|^{2}+\left|E_{i y}\right|^{2}+\left|E_{i z}\right|^{2}\right)\right|_{z=z_{*}}}\right)^{1 / 2}$.

The results shown in Fig. 2 are obtained for the frequency ELF-VLF emission band $1-6 \mathrm{kHz}$ for different transversal wave vector components $k_{x}$ and $k_{y}$. The plots in Fig. 3 are obtained for the frequency $f=3.5 \mathrm{kHz}$ for different incident angles $\alpha_{x}$ and $\alpha_{y}$. Here $\alpha_{x}=\arctan \left(k_{x} / k_{z i}\right), \alpha_{y}=$ $\arctan \left(k_{y} / k_{z i}\right)$, and $k_{z i}$ is the $z$ component of the incident wave vector at the altitude $z_{*}=180 \mathrm{~km}$. Note that $R$ and $h$ are even functions of angle $\alpha_{x}$ for the fixed angle $\alpha_{y}$. The dominant wave polarization near the ground surface is righthand polarized (typical for whistler waves).

\section{Discussion}

We consider the problem of numerical solutions of a complete system of wave equations in the lower layers of the 

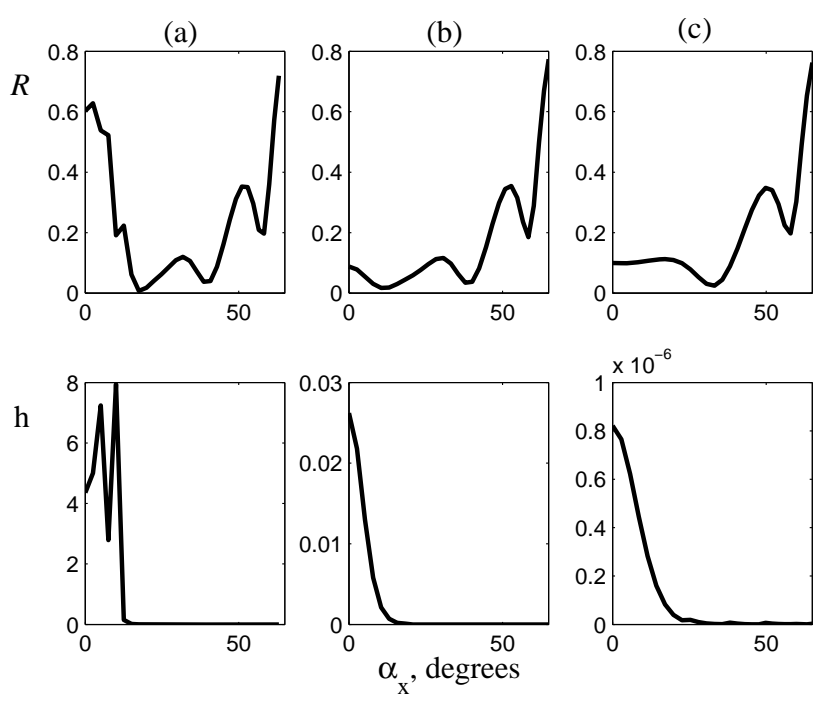

Figure 3. The energy reflection coefficient $R$ and the normalized amplitude of the horizontal magnetic field of the wave on the ground surface $h$ as functions of $\alpha_{x}$ : (a) $\alpha_{y}=0$, (b) $\alpha_{y}=17^{\circ}$, and (c) $\alpha_{y}=$ $34^{\circ}$.

nighttime ionosphere. To solve the system (Eqs. 4-6) with boundary conditions (Eqs. 7 and 9) at the altitudes $0 \leq z \leq$ $z_{*}$, we use a numerical method known as the boundary-value problem for ordinary differential equations. This method was examined by comparing the numerical and analytical results obtained in the previous paper by Bespalov and Mizonova (2004) for the case of a vertically propagating wave. The altitude $z_{*}$ was chosen for each frequency band and each value of the transverse wave vector, satisfying inequalities (11) and ranging from 125 to $220 \mathrm{~km}$. With the choice of the upper boundary $z=z_{*}$ the solution area covers a region that requires complete solution of the wave equations.

For typical conditions of the nighttime ionosphere, we find the energy reflection coefficient $R$ and the normalized amplitude of the horizontal magnetic field of the wave on the ground surface $h$ for the whistler wave incident from above. It can be seen in Figs. 2 and 3 that the value of $R$ and the amplitude of the wave magnetic field on the ground surface are sensitive enough to the wave parameters. For some frequencies, the value of $R$ has local maxima, which are especially notable in the case of a small angle of incidence. Possibly, the peaks of the function $R$ are caused by the resonance in the waveguide between the ground and the ionosphere. For example, in the case of normal and close-to-normal incidence of the wave, the function $R$ has local maxima at the frequencies $f=1.6,3.2$, and $4.9 \mathrm{kHz}$. These values correspond to the wavelengths in vacuums of 190,94 , and $61 \mathrm{~km}$. At the same time, the altitude where electron plasma frequency becomes equal to the wave frequency is on the order of 90$95 \mathrm{~km}$. Note that similar regularities are typical for the optical Fabry-Pérot resonator. We mention that this effect can appear if the wave can reach the ground surface. Taking into account the large value of the plasma refractive index above $100 \mathrm{~km}$, this is possible only if the angle of wave incidence on the ionosphere from above is small enough $\left(k_{\perp} c / \omega \leq 1\right)$.

Comparing the plots in Figs. 2 and 3 for different angles of wave incidence, we can show that the order of magnitude of $R$ is quite dependent on the area of strong wave refraction (that is the area below which the wave does not propagate). Indeed, if the transverse wave vector $k_{\perp}$ is less than the ratio $\omega / c$, then the parameter $R$ ranges from 0.4 to 0.8 (see Figs. 2a, b and 3a, b). If the transverse wave vector $k_{\perp}$ slightly exceeds the ratio $\omega / c$, then the wave reflects in the regions of strong attenuation $(z \sim 90-110 \mathrm{~km})$, and the value $R$ ranges from 0.02 to 0.4 (see Figs. 2c and 3 for the angles $\alpha_{x}<50^{\circ}$ ). If the wave vector significantly exceeds the ratio $\omega / c$, then the wave reflects upwards in the region of strong wave attenuation, and the value of $R$ is close to unity (see Figs. 2c and 3 for the angles $\alpha_{x}>50^{\circ}$ ).

The obtained results agree with the experimental data: good conditions for passage of the magnetospheric VLF emissions through the ionosphere exist for a low level of magnetic perturbation at the winter solar solstice (Manninen et al., 2014).

\section{Conclusions}

The problem of reflection and transmission of whistler waves from above in the low nighttime ionosphere has been considered. The solution of the wave equations for a horizontally stratified ionospheric plasma has been found by a numerical method known as the boundary-value problem. The area of the solution comprises a sharp boundary of the nighttime ionosphere $(\sim 100 \mathrm{~km})$ and the area of strong wave refraction and damping, which ranges from 125 to $220 \mathrm{~km}$ for different frequency bands and different transverse wave vectors. Frequency and incidence angle dependencies of the energy reflection coefficient $R$ and the normalized amplitude of the horizontal magnetic field of the wave on the ground surface $h$ have been calculated and analysed.

The value of $R$ is fairly dependent on the altitude of the wave reflection and can vary at the different angles of incidence from $1 / 1000$ to unity. In the cases of normal and closeto-normal wave incidence, the value $R$ has peaks, which correlate with peaks of the magnetic field on the ground surface. These peaks correspond to the resonance conditions in the waveguide between the ground surface and the sharp boundary of the low ionosphere.

The normalized amplitude of the horizontal magnetic field of the wave on the ground surface $h$ can be almost 1 order of magnitude greater than the electric field amplitude of the wave, which is incident from above on the lower nighttime ionosphere. This situation occurs at relatively small angles of incidence for the resonance waveguide frequencies. The electromagnetic wave near the ground surface is predomi- 
nantly right-hand polarized. Such a polarization is typical for whistler waves.

Data availability. The altitude dependencies of plasma density and electron-neutral collision frequency are publicly available at http: //omniweb.gsfc.nasa.gov/vitmo/iri_vitmo.html.

Competing interests. The authors declare that they have no conflict of interest.

Acknowledgements. Peter Bespalov was supported by RSF, project no. 16-12-10528 (Sects. 1 and 2) and the Russian Ministry of Science and Education, project no. 14.Z50.31.0007 (Sects. 3 and 4).

The topical editor, E. Roussos, thanks the one anonymous referee for help in evaluating this paper.

\section{References}

Bespalov, P. A. and Mizonova, V. G.: Reflection coefficient of whistler mode waves normally incident on the ionosphere, $\mathrm{Ge}$ omagn. Aeronomy, 44, 49-53, 2004.

Bilitza, D. and Reinisch, B.: International Reference Ionosphere 2007: Improvements and new parameters, J. Adv. Space Res., 42, 599-609, doi:10.1029/2007SW000359, 2008.

Budden, K. G.: The propagation of radio waves, Cambridge, London - New York, Cambridge University Press, 1985.

Galejs, J.: Terrestrial propagation of long electromagnetic waves, New York, Pergamon Press, 1972.
Ginzburg, V. L.: Propagation of electromagnetic waves in plasma, New York, Pergamon Press, 1970.

Helliwell, R. A.: Whistlers and related ionospheric phenomena, Stanford, Stanford University Press, 1965.

Kuzichev, I. V. and Shklyar, D. R.: On full-wave solution for VLF waves in the near-Earth space, J. Atmos. Sol.-Terr. Phy., 72, 1044-1056, doi:10.1016/j.jastp.2010.06.008, 2010.

Lehtinen, N. G. and Inan, U. S.: Radiation of ELF/VLF waves by harmonically varying currents into a stratified ionosphere with application to radiation by a modulated electrojet, J. Geophys. Res., 113, A06301, doi:10.1029/2007JA012911, 2008.

Manninen, J., Kleimenova, N. G., Fedorenko, Yu. V., Bespalov, P. A., and Turunen, T.: New results of structured VLF emissions observed simultaneously at two closely located stations near $L \sim 5.5$, Ann. Geophys., 32, 1163-1167, doi:10.5194/angeo-321163-2014, 2014.

Nygre'n, T.: A method of full wave analysis with improved stability, Planet. Space Sci., 30, 427-430, doi:10.1016/00320633(82)90048-4, 1982.

Pitteway, M. L. V. and Jespersen, J. L.: A numerical study of the excitation, internal reflection and limiting polarization of whistler waves in the lower ionosphere, J. Atmos. Terr. Phys., 28, 17-43, doi:10.1016/0021-9169(66)90004-3, 1966.

Shafranov, V. D.: Electromagnetic waves in a plasma, Reviews of Plasma Physics, edited by: Leontovich, M. A., NewYork, Consultants Bureau, 3, 2-154, 1967.

Storey, L. R. O.: An investigation of whistling atmospherics, Philos. T. R. Soc. S.-A, 246, 113-141, doi:10.1098/rsta.1953.0011, 1953.

Yagitani, S., Nagano, I., Miyamura, K., and Kimura, I.: Full wave calculation of ELF/VLF propagation from a dipole source located in the lower ionosphere, Radio Sci., 29, 39-54, doi:10.1029/93RS01728, 1994. 